ARTICLE OPEN

\title{
QTL-seq for the identification of candidate genes for days to flowering and leaf shape in pigeonpea
}

\author{
Vikas Singh ${ }^{1,2,6}$, Pallavi Sinha ${ }^{1,2,6}$, Jimmy Obala ${ }^{1,3,6}$, Aamir W. Khan ${ }^{1}$, Annapurna Chitikineni ${ }^{1}$, Rachit K. Saxena (iD ${ }^{1,4}$ and \\ Rajeev K. Varshney (iD ${ }^{1,5 \bowtie}$ \\ (c) The Author(s) 2021
}

To identify genomic segments associated with days to flowering (DF) and leaf shape in pigeonpea, QTL-seq approach has been used in the present study. Genome-wide SNP profiling of extreme phenotypic bulks was conducted for both the traits from the segregating population $\left(F_{2}\right)$ derived from the cross combination- ICP $5529 \times$ ICP 11605 . A total of 126.63 million paired-end (PE) whole-genome resequencing data were generated for five samples, including one parent ICP 5529 (obcordate leaf and lateflowering plant), early and late flowering pools (EF and LF) and obcordate and lanceolate leaf shape pools (OLF and LLS). The QTLseq identified two significant genomic regions, one on CLLO3 (1.58 Mb region spanned from 19.22 to $20.80 \mathrm{Mb}$ interval) for days to flowering (LF and EF pools) and another on CCLG08 (2.19 Mb region spanned from 6.69 to $8.88 \mathrm{Mb}$ interval) for OLF and LLF pools, respectively. Analysis of genomic regions associated SNPs with days to flowering and leaf shape revealed 5 genic SNPs present in the unique regions. The identified genomic regions for days to flowering were also validated with the genotyping-by-sequencing based classical QTL mapping method. A comparative analysis of the identified seven genes associated with days to flowering on 12 Fabaceae genomes, showed synteny with 9 genomes. A total of 153 genes were identified through the synteny analysis ranging from 13 to 36 . This study demonstrates the usefulness of QTL-seq approach in precise identification of candidate gene(s) for days to flowering and leaf shape which can be deployed for pigeonpea improvement.

Heredity (2022) 128:411-419; https://doi.org/10.1038/s41437-021-00486-x

\section{INTRODUCTION}

Pigeonpea [Cajanus cajan (L.)] is a protein-rich food legume that serves the dietary needs of more than a billion people in the developing world (Valenzuela 2011). Multiple uses of pigeonpea as food, livestock feed/fodder and domestic firewood make it a sustainable crop of small-holding farmers in the marginal and riskprone rainfed conditions (Saxena 2008). Development and adoption of improved varieties with higher yield will enhance the availability of plant-based protein for per capita consumption, thereby reducing the number of malnourished people across the world, especially in developing countries.

In pigeonpea, genomics approaches are being deployed to identify genomic regions that confer resistance/tolerance for different stresses. Both biparental mapping and association mapping approaches have been utilized to dissect complex traits in pigeonpea (Bohra et al. 2020). With the advantage of Next Generation Sequencing (NGS) technologies and availability of the pigeonpea reference genome sequence (Varshney et al. 2012), trait mapping approaches have mainly focused on mapping biotic stresses like sterility mosaic disease (SMD) and Fusarium wilt (FW) (Singh et al. 2016a, 2016b; Singh et al. 2017b; Saxena et al. 2017a; 2017b), abiotic stress like drought (Sinha et al. 2015), a marker for A4-derived CMS (Sinha et al. 2016), growth habit (Saxena et al. 2017c), A4-CMS restoration (Saxena et al. 2018), cleistogamous flower, shriveled seed and seed size (Yadav et al. 2019), seed protein content (Obala et al. 2019; Obala et al. 2020).

For developing new plant types that can suit various production niches, crop diversification with the development of photoinsensitive early maturing pigeonpea cultivars is a prerequisite (Saxena et al. 2019a). The first spontaneous mutant early maturing cultivar was detected in 1953 in a farmer's field. This triggered breeding of early maturing varieties, and subsequently, pigeonpea cultivars varying in maturity periods were bred (Saxena et al. 2019b). Since then, several early maturing cultivars have been bred in different parts of the world. Similarly, leaf shape is another morphological marker (naked eye polymorphism) in cytoplasmic male sterile (CMS) and the corresponding maintainer lines to track purity of the inbred lines and corresponding hybrids for large scale commercial hybrid seed production (Saxena et al. 2011b). It was noted that the obcordate leaf morphological marker is present in accession ICP 5529, which can be easily assessed visually in about 6 weeks after sowing.

Availability of pigeonpea genome assembly along with advances in NGS provides an opportunity to develop genomics tools and technologies for the mapping of agronomically important traits such as days to first flowering and leaf shape in

\footnotetext{
${ }^{1}$ Center of Excellence in Genomics \& Systems Biology, International Crops Research Institute for the Semi-Arid Tropics (ICRISAT), Hyderabad 502 324, India. ${ }^{2}$ International Rice Research Institute (IRRI), South-Asia Hub, ICRISAT, Hyderabad, India. ${ }^{3}$ Department of Science, Lira University, Lira, Uganda. ${ }^{4}$ Gujarat Biotechnology University (GBU), Gandhinagar 382011, Gujarat, India. ${ }^{5}$ State Agricultural Biotechnology Centre, Centre for Crop and Food Innovation, Food Futures Institute, Murdoch University, Murdoch, WA WA6150, Australia. ${ }^{6}$ These authors contributed equally: Vikas Singh, Pallavi Sinha, Jimmy Obala. Guest editor: Professor Reyazul Mir. ${ }^{凶}$ email: r.k.varshney@cgiar.org
} 
412

pigeonpea. QTL identification using whole-genome resequencing of two DNA bulks of progeny showing extreme phenotype (QTLseq) is an emerging technology that enables locating and refining candidate genomic regions more efficiently compared to traditional QTL mapping approaches (Takagi et al. 2013). As the QTLseq technique is independent of DNA marker development and genotyping the whole population, it is a time-saving and costeffective procedure as compared to the conventional QTL analysis. The QTL-seq approach has a wide applicability in QTL identification in many agronomically important crops like rice (Takagi et al. 2013), chickpea (Das et al. 2015; Singh et al. 2016b), groundnut (Pandey et al. 2017; Kumar et al. 2020), pigeonpea (Singh et al. 2016a; Singh et al. 2017b), cucumber (Lu et al. 2014), and tomato (Illa-Berenguer et al. 2015).

With the objective of identifying candidate genomic regions responsible for days to flowering (DF) and leaf shape in pigeonpea, QTL-seq approach was adopted. We were able to precisely localize genomic regions for two target traits and identify nine genic SNPs in seven candidate genes for DF and 39 genic SNPs in 20 candidate genes for leaf shape through QTL-seq approach. The involvement of candidate genes was further validated through co-segregation analysis in the entire $F_{2}$ population derived from ICP $5529 \times$ ICP 11605 through the genotyping-by-sequencing (GBS) based approach.

\section{MATERIALS AND METHODS}

Plant materials

One $F_{2}$ segregating population comprising 179 lines developed from a late duration (105 DF) and obcordate leaf shape, ICP 5529 and an early duration (67 DF) lanceolate leaf (normal) genotype, ICP 11605 was used in the present study (Obala et al. 2019). For trait evaluation, the parents and seeds of the mapping population were sown under field conditions. Sowing was done in $4 \mathrm{~m}$ long rows spaced $75 \mathrm{~cm}$ apart and $30 \mathrm{~cm}$ plant to plant distance within a row. Plot sizes were two rows for each of the two parents and $25-28$ rows in the $F_{2}$ s. All cultural practices were carried out. Days to flowering (DF) were recorded for individual plants as number of days to first flowering after sowing, whereas leaf shape data was recorded 6 weeks after sowing.

\section{Construction of pools}

Extreme bulks were prepared for days to first flowering and leaf shape traits based on precise phenotyping data obtained for $F_{2}$ population. For developing the extreme bulks for each trait, $15 \mathrm{~F}_{2} \mathrm{~S}$ with high mean phenotypic values and $15 \mathrm{~F}_{2} \mathrm{~S}$ with low mean phenotypic values were selected in the case of days to first flowering. For leaf shape, $15 \mathrm{~F}_{2} \mathrm{~S}$ of lanceolate leaf shape (LLS) (normal leaf) and $15 \mathrm{~F}_{2} \mathrm{~S}$ with obcordate leaf shape were selected for the preparation of the bulks. The equimolar concentration of DNA from $15 \mathrm{~F}_{2} \mathrm{~s}$ with high mean phenotypic values were pooled together as one bulk, and similarly, DNA from low mean phenotypic values were pooled together as another bulk. Thus, two bulks of pooled DNA each for both traits (DF and leaf shape) were used for library preparation and sequencing.

\section{Construction of libraries and Illumina sequencing}

A total of five genomic libraries (four from extreme bulks mentioned above and one from ICP 5529 parent) were prepared using TruSeq DNA Sample Prep kit LT, (set A) FC-121-2001. Two microgram of DNA from each sample was sheared using diagenode Bioruptor ${ }^{\circ}$ NGS, end-repaired and adapterligated. Size selection of libraries was performed using $2 \%$ agarose gel to get a target insert size of $500-600 \mathrm{bp}$ and purified for further analysis. Further, the libraries were enriched using adaptor compatible PCR primers. The size distribution of amplified DNA libraries was checked on an Agilent Technologies 2100 Bioanalyzer using a High Sensitivity chip. The DNA libraries were sequenced on Illumina HiSeq platform with HiSeq Reagent Kit v2 (500-cycles) to generate 250 base paired-end (PE) reads.

\section{Construction of reference-guided assembly}

The statistics of generated sequencing reads were estimated using the raspberry tool of NGS-QCbox (Katta et al. 2015). Furthermore, QTL-seq pipeline (http://genome-e.ibrc.or.jp/home/bioinformatics-team/mutmap, developed by Iwate Biotechnology Research Center, Japan) was used for calculating SNP-index. Briefly, the cleaned reads of ICP 5529 were first aligned to the reference genome (Varshney et al. 2012) using inbuilt BWA aligner (Li and Durbin 2009). Coval was used for post-processing and filtering of the alignment files (Kosugi et al. 2013). The variants called for ICP 5529 were then used to develop a reference-guided assembly of ICP 5529 by substituting the bases with confidence variants called in the genome. The reads from extreme bulks for both the traits were then aligned, and variants were called for both the bulks against the developed assembly.

\section{Calculation of SNP-index}

SNP-index for each SNP position was calculated for both the bulks as per Abe et al. (2012) using the formula: SNP-index (at a position) = Count of alternate base/ Count of reads aligned. The positions with read depth $<7$ in both the bulks and SNP-index $<0.3$ in either of the bulks were filtered out, and SNPs with homozygous alleles in both the bulks were used for $\triangle$ SNP-index calculation. $\triangle$ SNP-index can be calculated by subtracting the SNP-index of low bulk from SNP-index of high bulk. Only SNP positions with $\triangle S N P$-index $=-1$ (i.e., the allele called in high trait value-bulk was the same as that of the resistant parent while contrastingly different in low trait value-bulk) were considered as the causal SNPs responsible for the trait of interest. The possible effects of the identified SNPs were inferred using SnpEff v3.0 open-source program (Cingolani et al. 2012).

\section{RESULTS}

\section{Construction of extreme bulks for days to flowering and leaf shape}

Based on phenotyping data generated on $\mathrm{F}_{2} \mathrm{~S}$ derived from a crossing combination ICP $5529 \times$ ICP 11605 , two extreme bulks each for DF (early and late) and leaf shape (normal lanceolate and obcordate types) were prepared and subjected to the QTL-seq pipeline as shown in Figure S1 and Table S1. Phenotyping data on $\mathrm{DF}$ in $\mathrm{F}_{2} \mathrm{~S}$ showed a variation from 65 to 102 days in comparison to parental lines (105 days of ICP 5529 and 67 days of ICP 11605). The absolute difference between the parental lines was 38 days. Shapiro-Wilk test showed that distribution for DF was significantly different from a Gaussian distribution $(P \leq 0.05)$ (Table S2).

In the case of leaf shape trait, all the $F_{1} s$ from the cross had normal lanceolate leaves suggesting recessive nature of the obcordate leaf shape, while in the $F_{2}$ population, segregation for the same trait fitted well to the expected ratio of 3:1 (3 lanceolate: 1 obcordate leaf) (Table S3). Based on the phenotyping data, 15 $F_{2} S$ with early DF (65-66 days, early flowering pool, EF) and $15 F_{2} S$ with late DF (92-102 days, late-flowering pool, LF) were selected to prepare two extreme bulks (Table S4). For leaf shape $15 \mathrm{~F}_{2} \mathrm{~S}$ with LLS (lanceolate leaf pool, LLS pool) and $15 \mathrm{~F}_{2} \mathrm{~S}$ with obcordate leaf shape (obcordate leaf shape pool, OLS pool) were selected to prepare extreme bulks (Table S5).

\section{Whole genome sequencing and mapping of reads}

Five genomic libraries (two for DF bulks, two for leaf shape bulks, and one for ICP 5529, the obcordate leaf shape parent) were constructed and subjected to whole genome sequencing using Illumina HiSeq2500. In total, 142.80 million PE reads for DF extreme bulks ( 66.34 million reads for EF and 76.46 million reads for late-flowering bulks, respectively) and 122.74 million PE reads ( 60.86 for obcordate leaf and 61.88 million reads for lanceolate leaf bulks, respectively) for leaf shape were generated. A total of 58.63 million PE reads were generated for ICP 5529 (Table 1). Alignment of the PE reads generated from ICP 5529 to the reference genome assembly of pigeonpea (Varshney et al. 2012) resulted in an average depth of $9.15 \mathrm{X}$ and $90.70 \%$ genome coverage, allowing us to develop a reference-guided assembly of ICP 5529 (hereafter designated as ICP 5529 assembly).

Mapping of the PE reads generated from extreme bulks to the developed ICP 5529 assembly for DF resulted in 15.08X and 15.10X 
Table 1. Sequencing details of parental line and bulks and mapping of sequence reads.

\begin{tabular}{lllllll} 
Sample & $\begin{array}{l}\text { Number of } \\
\text { lines bulked }\end{array}$ & $\begin{array}{l}\text { Total reads } \\
\text { generated } \\
\text { (Million reads) }\end{array}$ & $\begin{array}{l}\text { High quality reads } \\
\text { (Million reads) }\end{array}$ & $\begin{array}{l}\text { Reads mapped } \\
\text { (Million reads) }\end{array}$ & $\begin{array}{l}\text { Genome } \\
\text { coverage at 1X }\end{array}$ \\
\hline ICP $5529^{\text {a }}$ & & 58.63 & 35.33 & 15.33 & $90.70 \%$ \\
depth (X)
\end{tabular}

EF Early flowering pool, LF Late flowering pool, OLS Obcordate leaf shape pool, LLS Lanceolate leaf shape pool.

aICP 5529 short reads were aligned to the publicly available pigeonpea genome of Asha (Varshney et al. 2012).

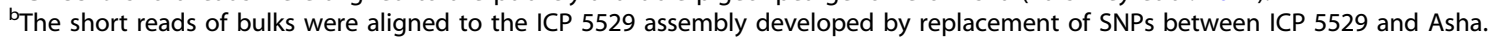

(a)

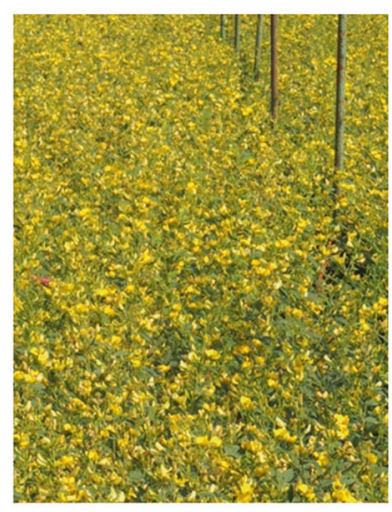

ICP 11605

Early flowering parent

(b)

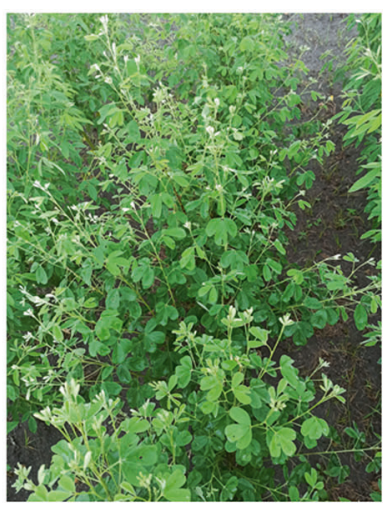

ICC 5529

Late flowering parent (c)

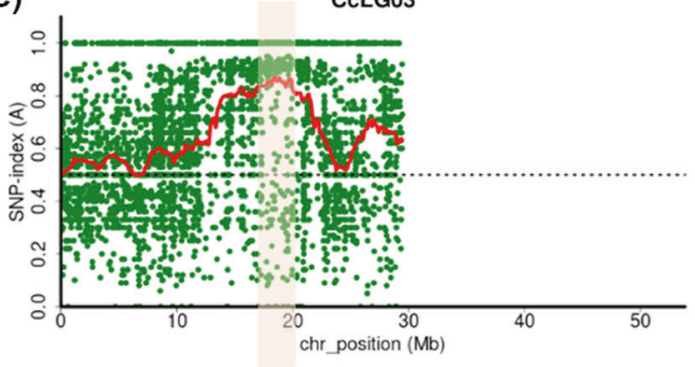

SNP-index plot of early flowering (EF) DNA pool

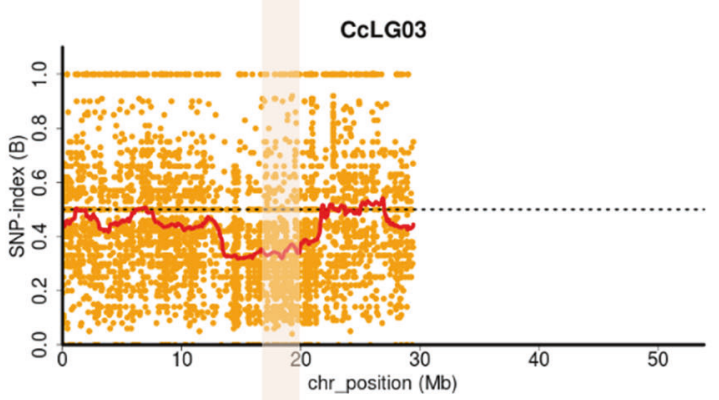

SNP-index plot of late flowering (LF) DNA pool

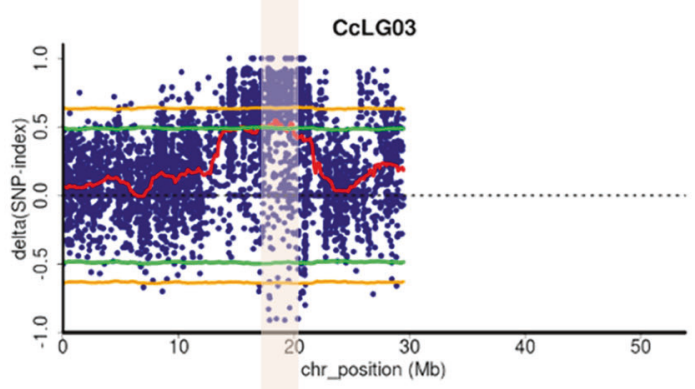

$\Delta($ SNP-index $)$ plot $=$ SNP-index EF- SNP-index LF

Fig. 1 A QTL-seq approach to identify genomic regions controlling days to flowering in pigeonpea. a ICP 11605: early flowering parent; b ICP 5529: late flowering parent; c SNP index plot between early flowering pool (top), late flowering pool (middle) and $\triangle$ SNP index plot (bottom) of chromosome CLG03 with statistical confidence interval under the null hypothesis of no QTLs (orange, $P<0.01$; and green, $P<0.05)$. The significant genomic region identified for days to flowering is shaded (1.58 Mb region spanned through $19.22-20.80 \mathrm{Mb})$.

sequencing depth and 93.26 and $93.13 \%$ coverage for late flowering (LF) and EF bulks, respectively. Similarly, for obcordate (OLS) and LLS bulks, we obtained alignment of $13.39 \mathrm{X}$ and $13.41 \mathrm{X}$ sequencing depth and 92.93 and $92.95 \%$ coverage, to the ICP
5529 assembly, respectively. The sequence alignment of LF bulk to the ICP 5529 assembly has provided 47,429 polymorphic SNPs. In this set, 9238 SNPs were homozygous in LF bulk. (Table S6). Similarly, of 46,510 identified SNPs between EF bulk and ICP 5529 
assembly and 7427 were homozygous in EF bulk. In the case of leaf shape, 10,521 were homozygous from the total identified 54,556 SNPs for OLS bulk and 8770 were homozygous from total 51,658 SNPs for LLS bulk after mapping the bulk reads on to the ICP 5529 assembly (Table S7).

\section{Candidate genomic region(s) for days to flowering}

QTL-seq analysis for DF revealed a genomic region on C $\mathrm{CLG03}$ (Fig. 1, Table S8 and Figs S2-S4). A genomic region spanning $1.58 \mathrm{Mb}(19.22-20.80 \mathrm{Mb})$ on CCLG03 showing significant $(P<0.05)$ deviation from equal inheritance of the two parental genomes had 56 SNPs with $\triangle$ SNP-index $=-1$ (Table S8). Of these 56 SNPs, nine SNPs were present in seven putative genes (Table 2). Of these nine genic SNPs, seven were in the intronic region and two SNPs were predicted in the exon regions of gene C.cajan_09900 and C. cajan_10078, associated with pentatricopeptide repeat-containing protein and cell division protein, respectively. Substitution of SNPs in the predicted gene C.cajan_09900 causing a non-synonymous substitution from Cysteine (aCg in ICP 5529 and LF pool) to Threonine (aTg in EF pool). Whereas, synonymous substitution in the predicted gene C.cajan_10078 were observed between the pools.

\section{Candidate genomic region(s) for leaf shape}

Sequence analysis of lanceolate and obcordate leaf bulks revealed a candidate genomic region on CcLG08 (Fig. 2, Table S9 and Figs. S5$\mathrm{S7})$. The genomic region spanning $2.19 \mathrm{Mb}$ region $(6.69-8.88 \mathrm{Mb})$ on CcLG08 revealed 210 SNPs with $\triangle \mathrm{SNP}$-index $=-1$, suggesting a biased inheritance of parental genomes in the two bulks (Table S9). The obcordate leaf bulk showed SNP-index $=0$ indicating that obcordate alleles were inherited from the obcordate leaf parent ICP 5529. By contrast, lanceolate leaf bulk at these 210 positions possesses SNP-index $=1$, indicating that their alleles were derived from the lanceolate leaf parent ICP 11605 (Table S9). Of the 210 SNPs, 39 SNPs were found in the genic regions of 20 genes. Of these 20 genic SNPs, 12 were present in the intronic region and eight SNPs were predicted in the exonic region of the genes (Table 3). SNP effect analysis of the eight exonic SNPs showed four synonymous and four non-synonymous substitutions. Substitution of SNPs in the predicted gene C.cajan_15991 and C.cajan_16002 causing a non-synonymous substitution from Threonine (Tgt/aTg in ICP 5529 and obcordate leaf pool) to Cysteine (Cgt/aCg in lanceolate leaf pool). Similarly, for two genes namely, C.cajan_16012 [Glycine (Gca in ICP 5529 and obcordate leaf pool) to Alanine (Aca in lanceolate leaf pool)] and C.cajan_16013 [Cysteine (cCt in ICP 5529 and obcordate leaf pool) to Threonine (cTt in lanceolate leaf pool) nsSNPs substitution was observed.

\section{DISCUSSION}

Advances in genomics have led to the development of various NGS based rapid trait mapping approaches like QTL-seq, MutMap, Indel-seq, BSA-Seq, etc (Varshney et al. 2020). NGS technologies have enabled modification and improvement of traditionally tricky, time-consuming bulked segregant analysis (BSA, Michelmore et al. 1991) into rapid and whole genome sequencing-based high-resolution trait mapping (Schlötterer et al. 2014). This approach has become popular nowadays due to affordable sequencing cost to many research groups and high throughput NGS tools. Moreover, the availability of draft genome sequence information in a species speeds up the sequencing of multiple individuals of that species and allows rapid identification of genomic variations as well as mapping and isolation of genes for causative mutations/target traits. Sequencing-based trait mapping combines both classical genetics and NGS platforms to map the associated traits. The application of sequencing-based trait mapping can be divided into two classes (i) trait mapping through bulk sequencing of populations, and (ii) trait mapping 
(a)

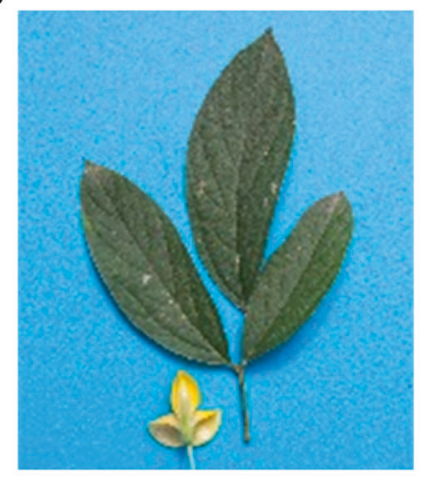

ICPL 11605

Lanceolate leaf parent

(b)

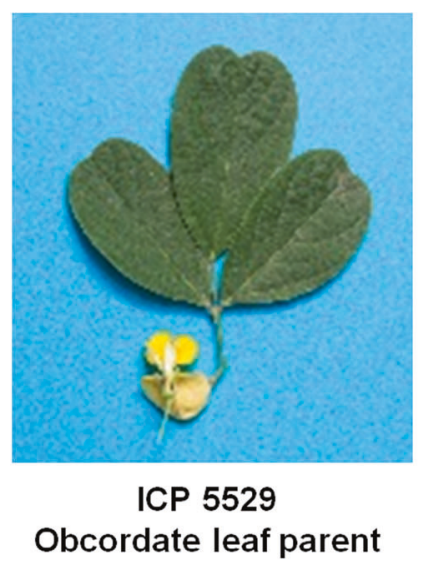

(c)

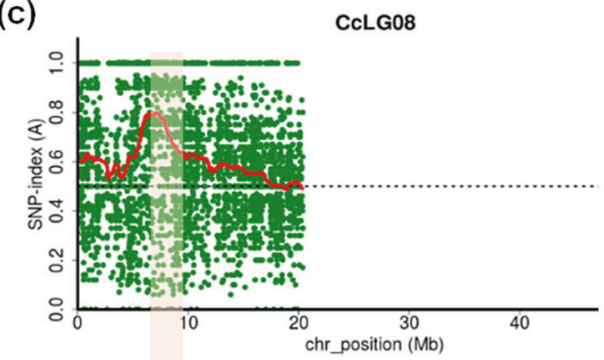

\section{SNP-index plot of lanceolate leaf shape (LLS) bulked DNA}

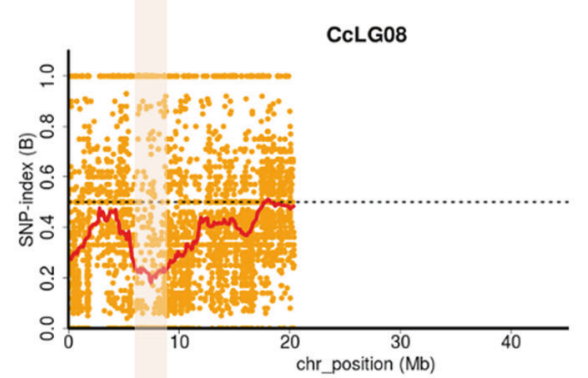

SNP-index plot of obcordate leaf shape (OLS) bulked DNA

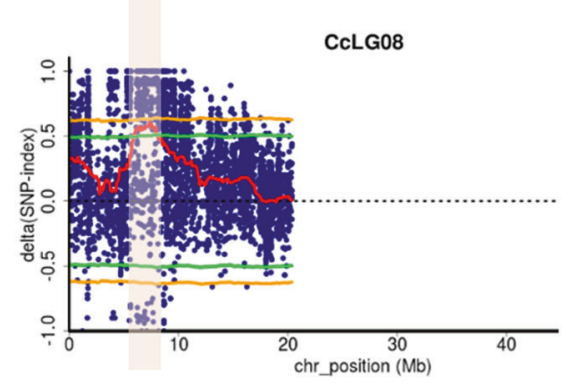

$\Delta($ SNP-index $)$ plot $=$ SNP-index LLS-SNP-index OLS

Fig. 2 A QTL-seq approach to identify genomic regions controlling obcordate leaf shape in pigeonpea. a ICP 11605: parent of lanceolate type leaf; $\mathbf{b}$ ICP 5529: parent of obcordate type leaf; c SNP index plot between lanceolate leaf shape DNA pool (top), obcordate leaf shape DNA pool (middle) and $\triangle$ SNP index plot (bottom) of chromosome CcLG08 with statistical confidence interval under the null hypothesis of no QTLs (orange, $P<0.01$; and green, $P<0.05$ ). The significant genomic region identified for obcordate leaf shape is shaded ( $2.18 \mathrm{Mb}$ region spanned from 6.69 to $8.88 \mathrm{Mb}$ ).

through complete sequencing of populations. Several examples of NGS-based trait mapping have been reported in many crop species (see Varshney et al. 2019).

\section{Genomic regions for leaf shape and days to flowering}

Genome sequencing of pigeonpea opened new avenues to enable sequencing-based trait mapping (Varshney et al. 2012). Sequencing-based bulked segregant analysis combined with nsSNPs substitution-based approach were utilized to map the candidate genes for FW and SMD resistance in pigeonpea (Singh et al. 2016a). Similar to SNP-based approach, Indel-seq approach (Indels based) was proposed and utilized for the first time toward the identification of candidate genomic regions/genes for FW and SMD resistance in pigeonpea (Singh et al. 2017b). In the present study, the sequencing of both bulks (EF and LF bulks) identified a comparatively large number of homozygous SNPs (9238 and 7427 SNPs for LF and EF, respectively). The identified SNPs were utilized to calculate the genome-wide SNP index information for both the pools. Analysis of SNPs located in the mapped candidate region on CcLG03 revealed a candidate gene, C.cajan_09900 coding for pentatricopeptide repeat (PPR) containing protein. It is well documented in the literature that PPR protein regulates flowering time in Arabidopsis (Emami et al. 2019). The role of C.cajan_09900 in the EF of pigeonpea can now be investigated further. We have also identified SNPs in the exonic regions of the gene $C$. cajan_10078, which codes for the cell division protein FtsZ homolog, however, a specific role of this gene in flowering not been reported. These two candidate genes especially gene $C$. cajan_09900 with non-synonymous mutation and few other genes discovered earlier in pigeonpea using candidate gene approach namely CCTFL1 and EARLY FLOWERING3 (Saxena et al. 2017c; Varshney et al. 2017) would be useful in expanding our understanding of molecular mechanism involved in flowering in pigeonpea and also in related legume species. Furthermore, sequence variations detected in these genes will facilitate the development of EF cultivars in pigeonpea through genomicsassisted breeding (Varshney et al. 2021). Flowering time in pigeonpea is critical as it directly correlates with the maturity of the plant. Nowadays as research efforts are being directed toward development of short duration or EF and early maturing pigeonpea varieties that mature around 100-120 days or early, the present findings will facilitate crop improvement programs. The short duration pigeonpea varieties will provide opportunities to include them in the existing cereal based cropping systems and 


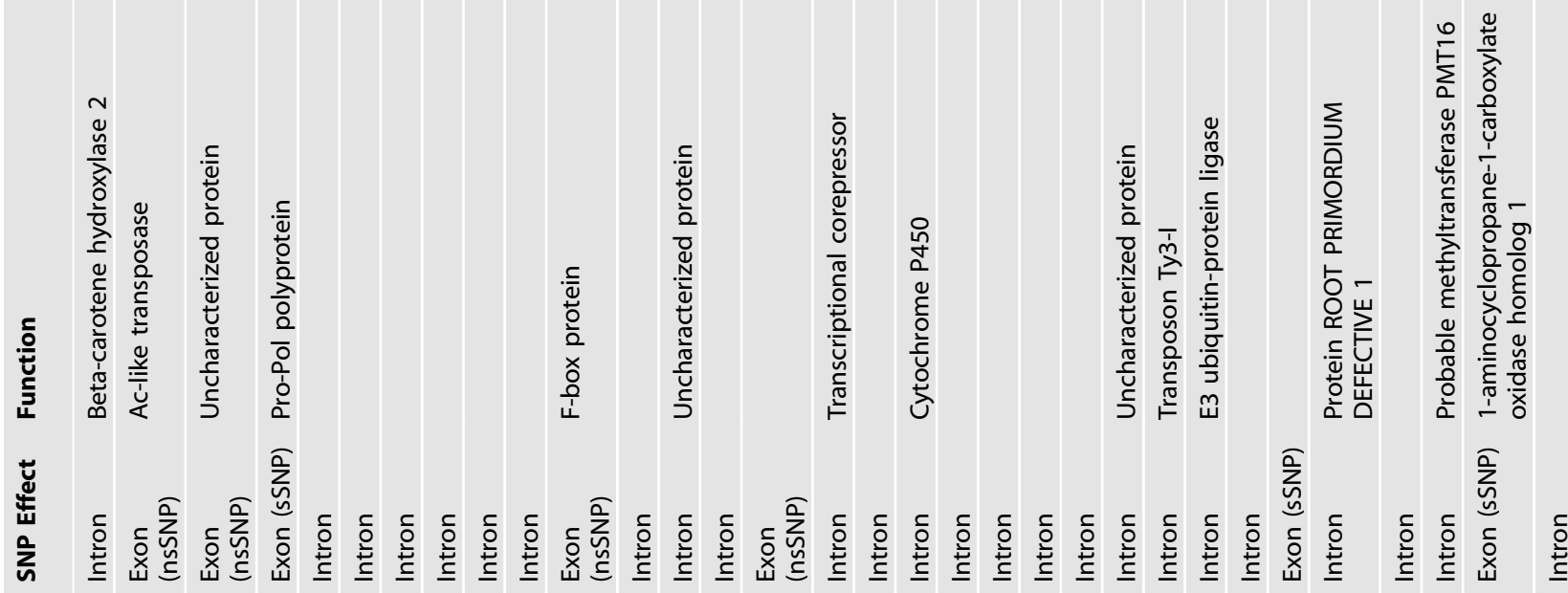

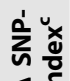

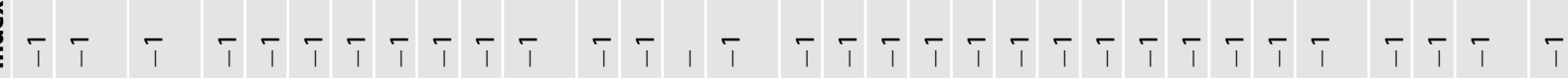

응

部当- - - - - - - - - - - - - - - - - - - - - -

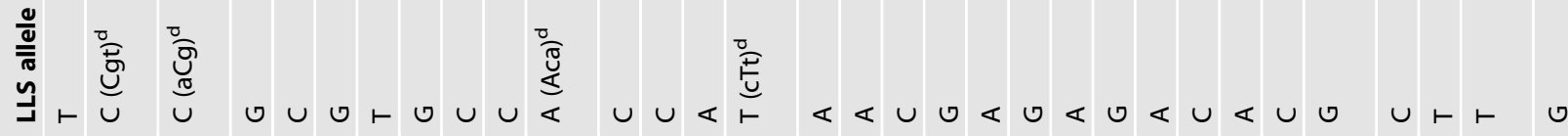

×

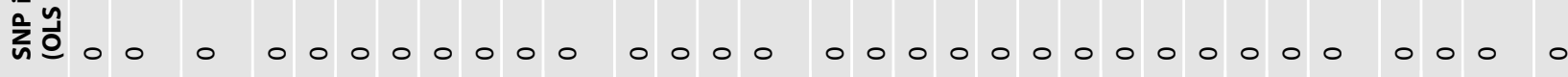

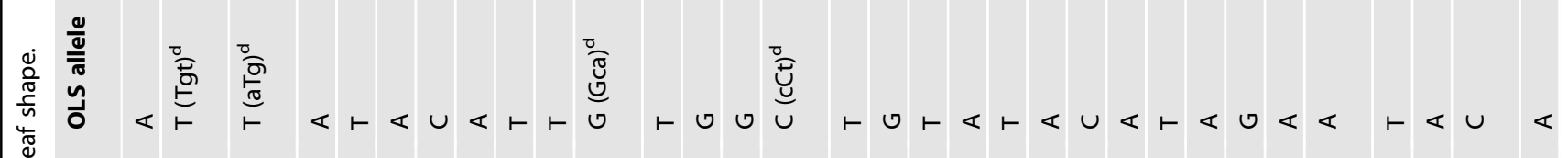

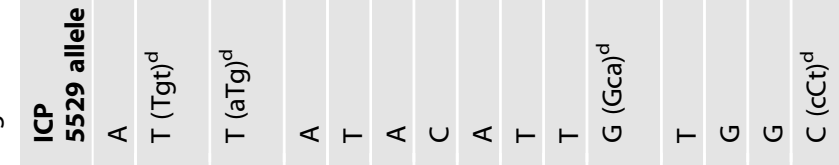

픙

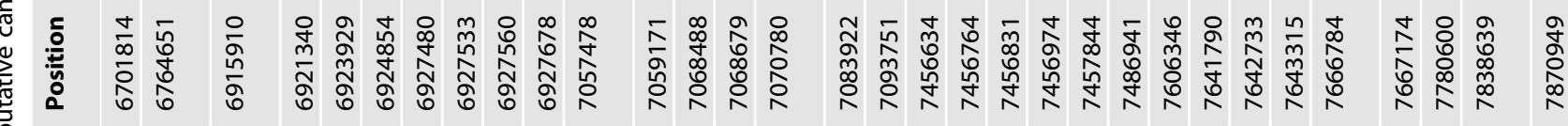

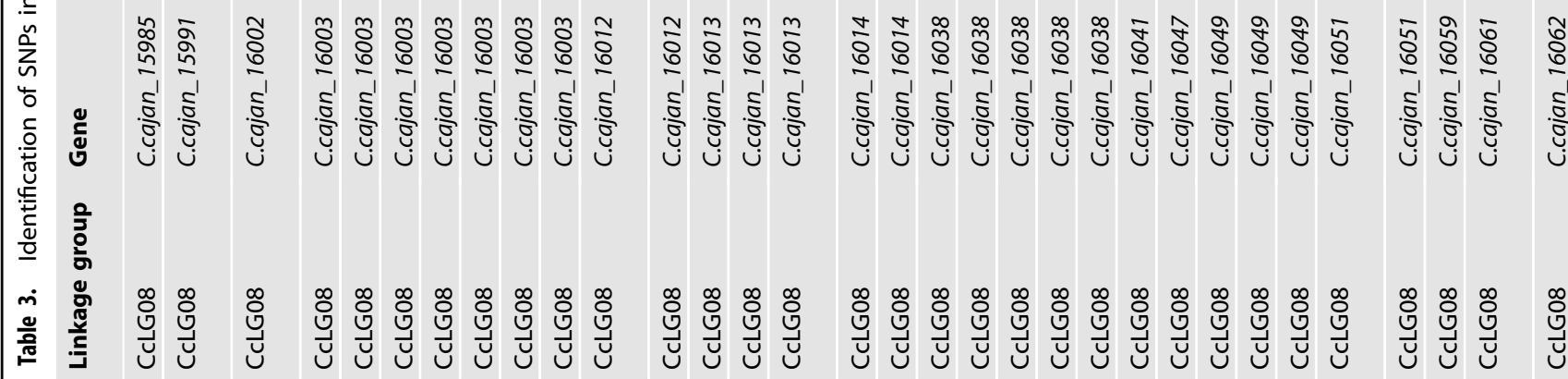




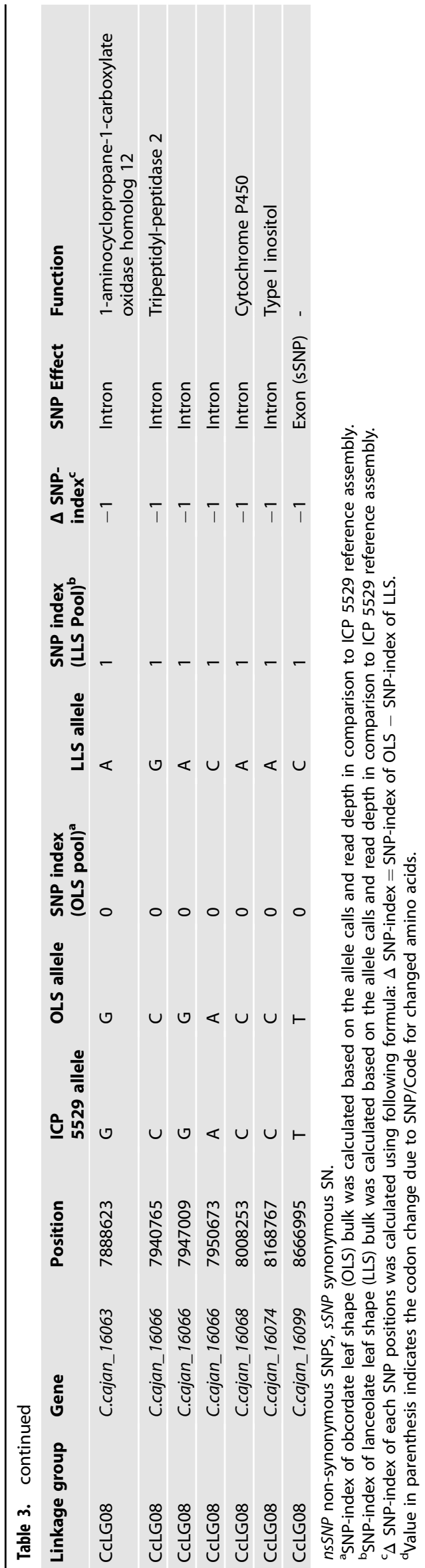

expand to new niches where pigeonpea could not be cultivated due to their LF or late maturity and photo-sensitivity.

Similarly, for obcordate leaf shape, a genomic region on CaLG08 was identified with four non-synonymous SNPs in four different genes (C.cajan_15991, C.cajan_16002, C.cajan_16012 and C. cajan_16013). Identified candidate gene C.cajan_15991 coding for Ac-like transposase has been earlier reported to play an important role in wrinkled shape character in pea (Bhattacharyya et al. 1990) and thus more understanding of this gene will be required to prove any possible role in the leaf shape of pigeonpea. We have also identified candidate gene C.cajan_16012, which codes for F-box protein. Previously, the role of F-box protein has been identified in leaf size and shape (Baute et al. 2017). Two identified candidate genes C.cajan_16002 and C.cajan_16013 have been reported as uncharacterized protein, thus more understanding and functional characterization will be required to understand their role in leaf shape development. These results highlighted the significance of the QTL-seq approach in identifying refined and reliable candidate regions for the traits of interest.

\section{Co-localization of genomic regions with QTLs identified through genetic linkage mapping approach}

Further, to validate our results obtained through QTL-seq approach, we have used available genetic map information on ICP $5529 \times$ ICP 11605 population (Table S10) (Obala et al. 2019). The phenotyping data obtained on entire population for DF were combined with the genetic map information to perform classical QTL analysis. Composite interval mapping identified a total of four QTLs for DF on CCLG03 with PVE ranging from 4.60 to $47.58 \%$ (Table S11). Three QTLs, namely qDF3.1 (39.58\%), qDF3.3 (47.58\%) and $q D F 3.4(16.18 \%)$ were identified as major effect QTLs and remaining one QTL showed minor effects ( $q D F 3.2$ ) for DF. All the four identified QTLs for DF were mapped between 16.68 and $22.23 \mathrm{Mb}(5.55 \mathrm{Mb})$ region on CcLG03. We have also identified the number of genes present within each QTL region and a minimum of 32 genes were identified within QTL, qDF3.3, while a maximum of 588 genes were identified within QTL, qDF3.1. All the four QTLs were found in these regions with different spans of the QTL window. Therefore, it is difficult to select the genes/genomic regions for molecular breeding without narrowing the candidate genomic regions. Interestingly, the QTL-seq approach also identified the DF related QTLs with a much narrow window (1.58 Mb region; $19.22-20.80 \mathrm{Mb}$ ) of the QTLs on CaLG03 (Table S12). Comparative to 588 coding genes reported in the conventional QTL approach, QTL-seq provided the opportunity to select the candidate genes from the identified two exonic SNPs. After validation of these identified exonic SNPs, these can be utilized in the crop improvement programs for the development of early maturing pigeonpea varieties.

\section{Comparative genetic mapping of days to flowering related genes across key legume crops}

To utilize the identified genomic regions in pigeonpea associated with DF, we tried to understand the relevance of the present study in other crops of Fabaceae family. We did a comparative mapping of the identified seven Cajanus cajan genes associated with DF with 12 crop genomes ((i) Arachis duranensis (Aradu), (ii) Arachis hypogaea (Arahy), (iii) Arachis ipaensis (Araip), (iv) Cicer arietinum, (v) Glycine max (Glyma), (vi) Glycine soja (Glyso), (vii) Lupinus angustifolius (Lupan), (viii) Medicago truncatula (Medtr), (ix) Phaseolus vulgaris (Phavu), ( $x$ ) Vigna angularis (Vigan), (xi), Vigna radiata (Vigra) (xii) Vigna unguiculata (Vigun).) (Fig. S8). We have performed protein blast using DELTA-BLASTP. Query coverage $>90 \%$, similarity $>60 \%$ and e-value $\leq 1 \mathrm{e}^{-10}$ were taken into consideration for best predicted results. Protein domain and gene functional analysis were performed using Interpro (Table S13). We have found two genes, earlier reported which plays an important role in flowering in Arabidopsis. The interpro domain 
prediction provided similar domain region hits in the protein structure of PPR domain C.cajan_09900 reflected Pentatricopeptide repeat in its protein structure. PRECOCIOUS1 (POCO1), a p-class PPR repeat protein reported as to affect flowering time in Arabidopsis thaliana (Emami et al. 2019). Another gene $C$. cajan_09938 hits the Zinc finger PHD-type protein named PHD finger domain containing protein (PFD) is identified to suppress the flowering in Arabidopsis thaliana (Yokoyama et al. 2019). Conserved genomic regions were identified with nine genomes out of the selected 12 targeted genomes. A high degree of conserved collinear synteny among the chromosome 6 of Aradu, Arahy and Araip were identified. On chromosome 3 of Cicer, chromosome 19 of Glyma and Glyso and chromosome 3, 1 and 7 of Vigra, Phavu and Medtr respectively, we have also found the similarity with the identified Cajanus cajan genes (Table S14). An interpretation in the view of domain search of candidate genes can give proportional understanding of functions. Comparative analysis of the seven Cajanus cajan genes with the 12 genomes, mapped to 153 homologous genes ranged from 13 (C. cajan_09900 and C.cajan_09958) to 36 (C.cajan_09965). The number of genes mapped to the other crop genomes ranged from 9 (Cicer areitinum and Vigna radiata) to 20 (Glycine max) (Table S15). Identified genes in the present study through synteny analysis in the other crops can be validated and utilized for the development of early duration crop varieties.

\section{CONCLUSION}

The present study has provided candidate genes for DF and leaf shape in pigeonpea using QTL-seq approach. Some candidate genes have been prioritized using information on nonsynonymous SNPs and homology based analysis. Moreover, we have also enriched the genomic regions associated with DF through GBS based QTL analysis. In summary, this study has provided the most promising candidate gene/s for DF (C. cajan_09900) and leaf shape (C.cajan_15991, C.cajan_16002, C. cajan_16012 and C.cajan_16013) in pigeonpea. Additionally, comparative genetic mapping of DF related genes with other crops, revealed several important genes in nine other Fabaceae crop genomes. These candidate genes will be helpful in identification, cloning and functional validation of causal mutation or gene/s responsible for DF and leaf shape in pigeonpea and other related legume species.

\section{DATA AVAILABILITY}

All the data generated in the present study is provided in the Supplementary Information and sequencing data deposited as Bioproject ID PRJNA774652 in NCBI.

\section{REFERENCES}

Abe A, Kosugi S, Yoshida K, Natsume S, Takagi H, Kanzaki H et al. (2012) Genome sequencing reveals agronomically important loci in rice using MutMap. Nat Biotechnol 30:174-178

Baute J, Polyn S, De Block J, Blomme J, Van Lijsebettens M, Inzé D (2017) F-Box Protein FBX92 affects leaf size in Arabidopsis thaliana. Plant Cell Physiol 58:962-975

Bhattacharyya MK, Smith AM, Ellis TH, Hedley C, Martin C (1990) The wrinkled-seed character of pea described by Mendel is caused by a transposon-like insertion in a gene encoding starch-branching enzyme. Cell 60:115-122

Bohra A, Saxena KB, Varshney RK, Saxena RK (2020) Genomics-assisted breeding for pigeonpea improvement. Theor Appl Genet 133:1721-1737

Cingolani P, Platts A, Wang le L, Coon M, Nguyen T, Wang L et al. (2012) A program for annotating and predicting the effects of single nucleotide polymorphisms, SnpEff: SNPs in the genome of Drosophila melanogaster strain w (1118); iso-2; iso-3. Fly (Austin) 6:80-92

Das S, Upadhyaya HD, Bajaj D, Kujur A, Badoni S, Laxmi Kumar V et al. (2015) Deploying QTL-seq for rapid delineation of a potential candidate gene underlying major trait-associated QTL in chickpea. DNA Res 22:193-203
Emami H, Kempken F, PRECOCIOUS1 (POCO1) (2019) A mitochondrial pentatricopeptide repeat protein affects flowering time in Arabidopsis thaliana. Plant J 100(2):265-278

Illa-Berenguer E, Van Houten J, Huang Z, van der Knaap E (2015) Rapid and reliabl identification of tomato fruit weight and locule number loci by QTL-seq. Theor Appl Genet 128:1329-1342

Katta MAVSK, Khan AW, Doddamani D, Thudi M, Varshney RK (2015) NGS-QCbox and raspberry for parallel, automated and rapid quality control analysis of largescale next generation sequencing (Illumina) data. PLoS ONE 10:e0139868

Kosugi S, Natsume S, Yoshida K, MacLean D, Cano L, Kamoun S et al. (2013) Coval: improving alignment quality and variant calling accuracy for next-generation sequencing data. PloS ONE 8:e75402

Kumar R, Janila P, Vishwakarma M, Khan AW, Manohar S, Gangurde SS et al. (2020) Whole genome re-sequencing-based QTL-seq identified candidate genes and molecular markers for fresh Seed dormancy in groundnut. Plant Biotechnol J 18:992-1003

Li H, Durbin R (2009) Fast and accurate short read alignment with Burrows-Wheeler transform. Bioinformatics 25:1754-1760

Lu H, Lin T, Klein J, Wang S, Qi J, Zhou Q et al. (2014) QTL-seq identifies an early flowering QTL located near Flowering Locus T in cucumber. Theor Appl Genet 127:1491-1499

Michelmore RW, Paran I, Kesseli RV (1991) Identification of markers linked to diseaseresistance genes by bulked segregant analysis - a rapid method to detect markers in specific genomic regions by using segregating populations. Proc Natl Acad Sci USA 88:9828-9832

Obala J, Saxena RK, Singh V, Sameer Kumar CV, Saxena KB, Tongoona P et al. (2019) Development of sequence-based markers for seed protein content in pigeonpea. Mol Gen Genom 294:57-68

Obala J, Saxena RK, Singh VK, Kale SM, Garg V, Sameer Kumar CV et al. (2020) Seed protein content and its relationships with agronomic traits in pigeonpea is controlled by both main and epistatic effects QTLs. Sci Rep 10:214

Pandey MK, Khan AW, Singh VK, Vishwakarma MK, Shasidhar Y, Kumar V et al. (2017) QTL-seq approach identified genomic regions and diagnostic markers for rust and late leaf spot resistance in groundnut (Arachis hypogaea L.). Plant Biotechnol J 15:927-941

Saxena KB (2008) Genetic improvement of pigeonpea-A review. Trop Plant Biol $1: 159-178$

Saxena KB, Choudhary AK, Srivastava RK, Bohra A, Saxena RK, Varshney RK (2019) Origin of early maturing pigeonpea germplasm and its impact on adaptation and cropping systems. Plant Breed 138:243-251

Saxena KB, Saxena RK, Hickey LT, Varshney RK (2019b) Can a speed breeding approach accelerate genetic gain in pigeonpea? Euphytica 215:202

Saxena KB, Vales MI, Kumar RV, Sultana R, Srivastava RK (2011b) Ensuring genetic purity of pigeonpea hybrids by incorporating a naked-eye polymorphic marker in A and B lines. Crop Sci 51:1564-1570

Saxena RK, Kale SM, Kumar V, Parupalli S, Joshi S, Singh VK et al. (2017a) Genotypingby-sequencing of three mapping populations for identification of candidate genomic regions for resistance to sterility mosaic disease in pigeonpea. Sci Rep 7:1813

Saxena RK, Obala J, Sinjushin A, Sameer Kumar CV, Saxena KB, Varshney RK (2017c) Characterization and mapping of Dt1 locus which co-segregates with CCTFL1 for growth habit in pigeonpea. Theor Appl Genet 130:1773-1784

Saxena RK, Patel K, Sameer Kumar CV, Tyagi K, Saxena KB, Varshney RK (2018) Molecular mapping and inheritance of restoration of fertility (Rf) in A4 hybrid system in pigeonpea (Cajanus cajan (L.) Millsp.). Theor Appl Genet 131:1605-1614

Saxena RK, Singh VK, Kale SM, Tathineni R, Parupalli S, Kumar V et al. (2017b) Construction of genotyping-by-sequencing based high-density genetic maps and QTL mapping for fusarium wilt resistance in pigeonpea. Sci Rep 7:1911

Schlötterer C, Tobler R, Kofler R, Nolte V (2014) Sequencing pools of individualsMining genome-wide polymorphism data without big funding. Nat Rev Genet 15:749-763

Singh VK, Khan AW, Saxena RK, Sinha P, Kale SM, Parupalli S et al. (2017b) Indel-seq: a fast-forward genetics approach for identification of trait-associated putative candidate genomic regions and its application in pigeonpea (Cajanus cajan). Plant Biotechnol J 15:906-914

Singh VK, Khan AW, Jaganathan D, Thudi M, Roorkiwal M, Takagi H et al. (2016a) QTLseq for rapid identification of candidate genes for 100-seed weight and root/ total plant dry weight ratio under rainfed conditions in chickpea. Plant Biotechnol J 14:2110-2119

Singh VK, Khan AW, Saxena RK, Kumar V, Kale SM, Sinha P et al. (2016b) Next generation sequencing for identification of candidate genes for Fusarium wilt and sterility mosaic disease in pigeonpea (Cajanus cajan). Plant Biotechnol J 4:1183-1194 
Sinha P, Pazhamala T, Singh VK, Saxena RK, Krishnamurthy L, Azam S et al. (2016) Identification and validation of selected universal stress protein domain containing drought-responsive genes in pigeonpea (Cajanus cajan L.). Front Plant Sci 6:1065

Sinha P, Saxena KB, Saxena RK, Singh VK, Suryanarayana V, Sameer Kumar V et al. (2015) Association of nad7a gene with cytoplasmic male sterility in pigeonpea. Plant Genome 8:1-12

Takagi H, Abe A, Yoshida K, Kosugi S, Natsume S, Mitsuoka C et al. (2013) QTL-seq: rapid mapping of quantitative trait loci in rice by whole genome resequencing of DNA from two bulked populations. Plant J 74:174-183

Valenzuela H (2011) Pigeon pea: a multipurpose crop for Hawaii, March-April-May edn. Hanai'Ai/The Food Provider, Hawaii, p. 1-8

Varshney RK, Chen W, Li Y, Bharti AK, Saxena RK, Schlueter JA et al. (2012) Draft genome sequence of pigeonpea (Cajanus cajan), an orphan legume crop of resource-poor farmers. Nat Biotechnol 30:83-89

Varshney RK, Pandey MK, Bohra A, Singh VK, Thudi M, Saxena RK (2019) Toward the sequence-based breeding in legumes in the post-genome sequencing era. Theor Appl Genet 132:797-816

Varshney RK, Sinha P, Singh VK, Kumar A, Zhang Q, Bennetzen JL (2020) 5Gs for crop genetic improvement. Curr Opin Plant Biol 56:190-196

Varshney RK, Bohra A, Yu J, Graner A, Zhang Q, Sorrells ME (2021) Designing future crops: Genomics-assisted breeding comes of age. Trends Plant Sci 26:631-649

Varshney RK, Saxena R, Upadhyaya H, Khan AW, Yu Y, Kim C, Rathore A et al. (2017) Whole-genome resequencing of 292 pigeonpea accessions identifies genomic regions associated with domestication and agronomic traits Nat Genet 49:1082-1088. https://doi.org/10.1038/ng.3872

Yadav P, Saxena KB, Hingane A, Kumar C, Kandalkar VS, Varshney RK et al. (2019) An "Axiom Cajanus SNP Array" based high density genetic map and QTL mapping for high-selfing flower and seed quality traits in pigeonpea. BMC Genom 20:235

Yokoyama Y, Kobayashi S, Kidou SI (2019) PHD type zinc finger protein PFP represses flowering by modulating FLC expression in Arabidopsis thaliana. Plant Growth Regul 88:49-59

\section{ACKNOWLEDGEMENTS}

The work was supported in parts by the United States Agency for International Development (USAID)- India Mission, Department of Agriculture and Cooperation, Ministry of Agriculture, Government of India and Bill \& Melinda Gates Foundation (grant number OPP1005131). This work has been undertaken as part of the CGIAR Research Program on Grain Legumes and Dry Land Cereals. ICRISAT is a member of CGIAR Consortium.

\section{AUTHOR CONTRIBUTIONS}

RKV was responsible for coordination of the study, also contributed in designing study, writing, analysis and interpretation. VKS was responsible for designing, generating data, analysis and writing. PS also contributed in designing, generating data, analysis and writing. JO contributed in generating phenotyping data, analysis and writing. AWK contributed to analysis of sequencing data and QTL-seq analysis. AC contributed in sequencing data generation and writing. RKS contributed in coordination of study, generation of segregating population, phenotyping, sequencing data generation, writing and interpretation.

\section{COMPETING INTERESTS}

The authors declare no competing interests.

\section{ADDITIONAL INFORMATION}

Supplementary information The online version contains supplementary material available at https://doi.org/10.1038/s41437-021-00486-x.

Correspondence and requests for materials should be addressed to Rajeev $\mathrm{K}$. Varshney.

Reprints and permission information is available at http://www.nature.com/reprints

Publisher's note Springer Nature remains neutral with regard to jurisdictional claims in published maps and institutional affiliations.

(C) Open Access This article is licensed under a Creative Commons Attribution 4.0 International License, which permits use, sharing, adaptation, distribution and reproduction in any medium or format, as long as you give appropriate credit to the original author(s) and the source, provide a link to the Creative Commons license, and indicate if changes were made. The images or other third party material in this article are included in the article's Creative Commons license, unless indicated otherwise in a credit line to the material. If material is not included in the article's Creative Commons license and your intended use is not permitted by statutory regulation or exceeds the permitted use, you will need to obtain permission directly from the copyright holder. To view a copy of this license, visit http://creativecommons. org/licenses/by/4.0/.

(c) The Author(s) 2021 